\title{
ОБРАЗОВАНИЕ УСТОИЧИВЫХ АНТИПРОВАЛОВ В НЕОДНОРОДНЫХ ПРИМЕСНЫХ СПЕКТРАХ ПОД ВОЗДЕЙСТИЕМ НЕРЕЗОНАНСНОГО ЛАЗЕРНОГО ОБЛУЧЕНИЯ
}

\author{
(Представил К. К. Ребане)
}

Фотохимическое выжигание провалов в спектрах примесных молекул в низкотемпературных твердотельных матрицах $\left[{ }^{1,2}\right]$ существенно расширило возможности спектроскопии таких систем, так как позволило осуществить высокодобротную селекцию примесных молекул по частоте электронного перехода. Само явление фотохимического выжигания провалов основывается на спектрально высокоселективном инициировании трансформации примесей монохроматическим светом. Как научный, так и практический интерес представляют и решение обратной задачи спектрально селективного торможения фотохимической реакции (фототрансформации примеси), и связанная с ним возможность формирования узких устойчивых антипровалов. Отметим, что узких (по сравнению с неоднородной полосой или линией) антипровалов не образуется в процессе спектрального перераспределения выжженных селективно молекул (фотопродукта), как правило, из-за неполной корреляции между частотами переходов исходной конформации примеси и фотопродукта $\left[{ }^{3-5}\right]$.

В настоящей работе сообщается о наблюдении образования узких устойчивых антипровалов в примесных спектрах возбуждения под воздействием узкополосного импульсного облучения вне полосы поглощения примеси. Обсуждаются возможный механизм явления и его спектроскопические применения.

Изучался твердый раствор перилена в этаноле с концентрацией примеси $10^{-4}$ моль/л, помещенный в плоскопараллельную кварцевую кювету толщиной 0,2 мм при $T=4,2 \mathrm{~K}$. Из-за неоднородного уширения чисто электронный $S_{1} \rightarrow S_{0}$-переход проявляется в спектре поглощения этой системы в виде бесструктурной полосы с максимумом при 439,5 нм и полушириной 5 нм. Спектры возбуждения измерялись с помощью импульсного лазера на красителе FL 2000, накачиваемого эксимерным лазером EMG 101. Параметры световых импульсов, попадающих на объект, были следующими: длительность импульса $10^{-8}$ с, спектральная ширина 0,04 нм, пиковая плотность возбуждения в импульсе $10 \mathrm{kBT} \cdot \mathrm{cm}^{-2}$, частота следования импульсов 10 Гц. Флуоресценция регистрировалась на спектрометре ДФС-24.

Результаты измерений приведены на рис. 1, где представлены спектры возбуждения $0^{\prime} \rightarrow 1 \quad\left(1379 \mathrm{~cm}^{-1}\right)$ линии флуоресценции $S_{1} \rightarrow S_{0^{-}}$ перехода, снятые при сканировании лазера на красителе в $0^{\prime} \leftarrow 0$-полосе поглощения $S_{1} \leftarrow S_{0}$-перехода от 441,6 до 442 нм и регистрации флуоресценции на длине волны 470,4 нм (ширина щелей монохроматора 0,65 нм). 
Спектры $a$ соответствуют исходной ситуации. Спектры 6 измерены при тех же условиях после облучения образца в течение $10^{3}$ с вне полосы поглощения на длинах волн 448,8 и 448,65 нм. Облучение осуществлялось тем же лазером на красителе, пиковая плотность возбуждения была порядка $100 \mathrm{MBT} \cdot \mathrm{cm}^{-2}$. В разностных спектрах $8=\sigma-a$ отчетливо

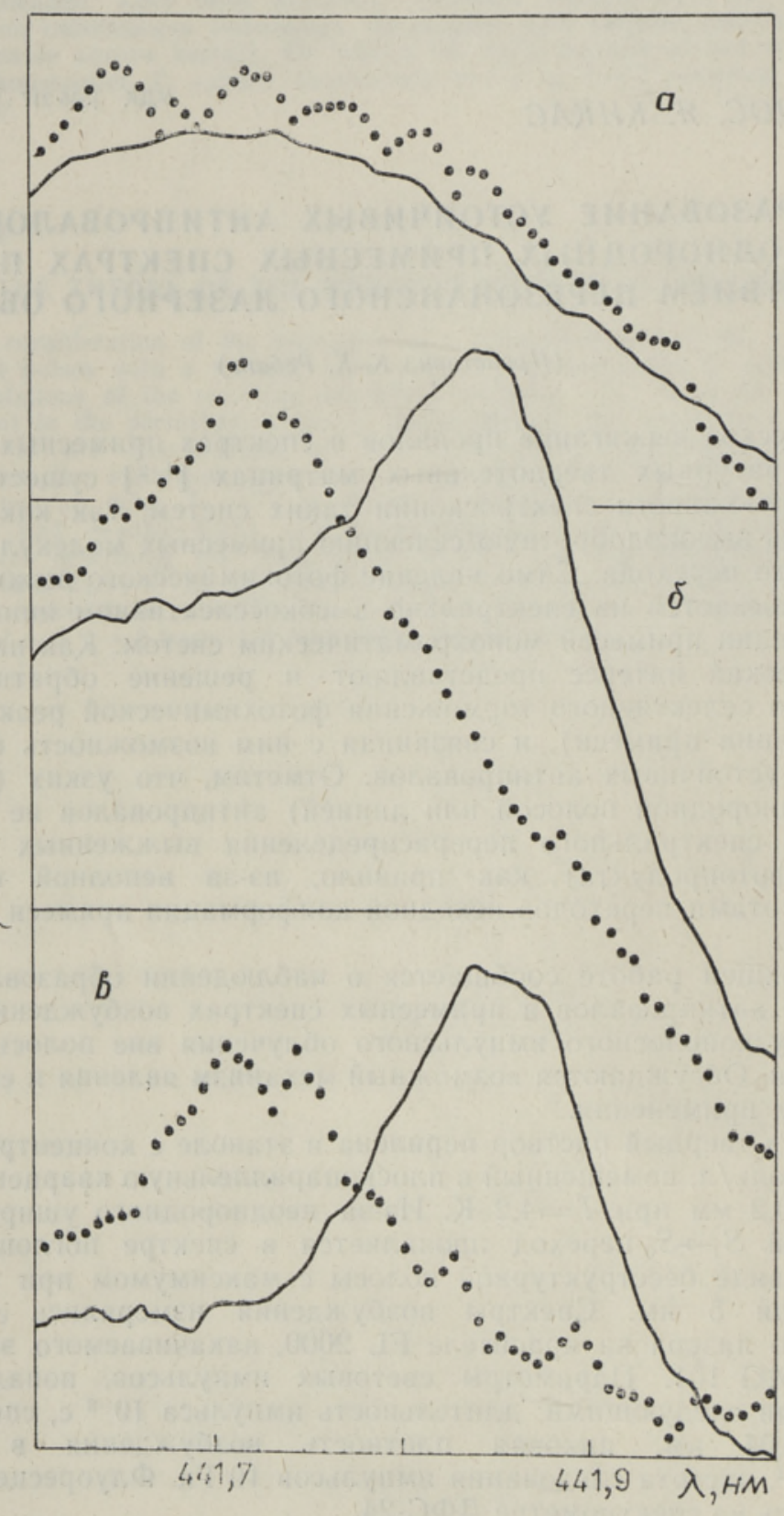

Рис. 1. Исходные спектры возбуждения $(a)$ и те же спектры после облучения образца на длинах волн 448,8 нм (сплошная кривая) и 448,65 нм (пунктирная кривая) вне полосы поглощения перилена в течение 15 мин. (б), а также разностные спектры $(B=\sigma-a)$. 
проявляется образование узкого (с полушириной 0,1 нм) антипровала, сдвиг которого совпадает со сдвигом длины волны нерезонансного длинноволнового облучения $(0,15$ нм).

Установление точного механизма такого явления требует дальнейших исследований. По-видимому, первой ступенью процесса является заселение $S_{1}$-состояния молекулы перилена, возможно, через двухквантовое возбуждение более высоких электронных состояний с последующей энергетической релаксацией (рис. 2). Как известно, прямое монохроматическое возбуждение $S_{1}$-состояния молекулы перилена в спиртовой матрице приводит к образованию устойчивых провалов в спектре $S_{1} \leftarrow S_{0}$-поглощения $\left[{ }^{2}\right]$. Образование таких провалов наблюдалось нами и при импульсном возбуждении (рис. $3, a$ ), несмотря на сравнительно низкую частоту повторения импульсов. Молекулярная природа фототрансформаций, приводящих к выжиганию провалов, точно не установлена, однако известно, что они фотообратимы и приводят к равномерному по неоднородной полосе перераспределению «выжженных» молекул

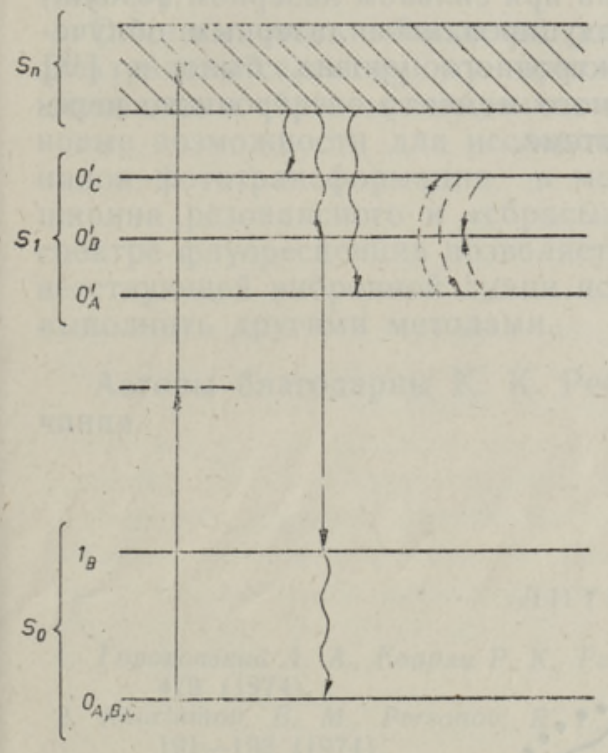

Рис. 2.

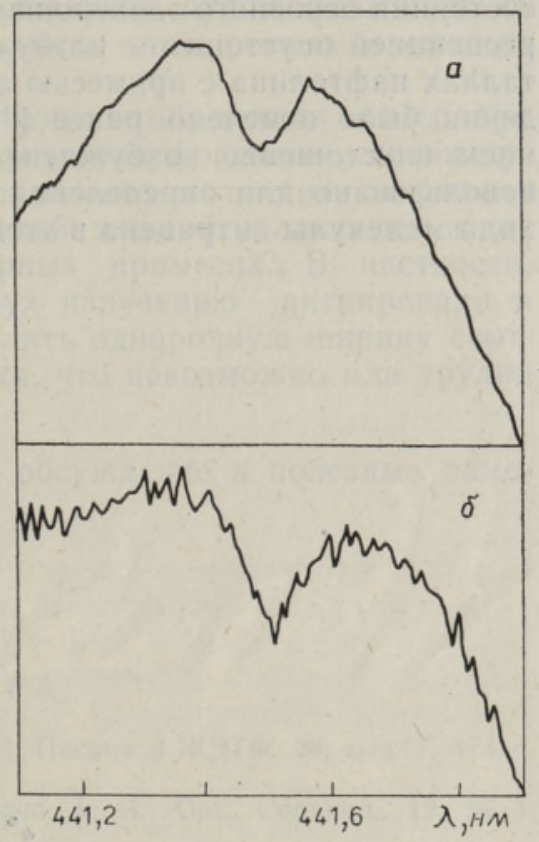

Рис. 3.

Рис. 2. Предполагаемая схема переходов при образовании антипровала. Прямыми стрелками указаны светоиндуцированные переходы, волнистыми - безызлучательная электронно-колебательная релаксация, штриховыми - взаимные фототрансформации примесей в $S_{1}$-состоянин. С помощью индексов $A, B, C$ из сплошного неоднородного распределения выделены три типа центров, отличающихся частотами чисто электронного и вибронного переходов. Только для центров типа $B$ выполнсно условие резонансного сбрасывання заселенности $S_{1}$-состояния. Вибронные уровни и спонтанные радиационные переходы для центров $A, C$ не указаны.

Рнс. 3. Устойчнвый провал в спектре возбуждения перилена (регистрация флуоресценции на длине волны $\lambda=470,1$ нм, $\Delta \lambda=0,65$ нм), выжженный облучением образца в течение 15 мин на длине волны 441,5 нм при пиковой интенсивности облучения порядка $10 \mathrm{MBT} \cdot \mathrm{cm}^{-2}(a)$, и динамический провал в спектре возбуждения (условия регистрации те же), возникший из-за индуцированного сброса заселенности $S_{1}$-состояния облучением образца на длине волны 488,5 нм при пиковой интенсивности $100 \mathrm{MBT}^{-c^{-2}}(б)$. 
(фотопродукта); узких (по сравнению с неоднородной полосой поглощения) антипровалов не наблюдается $\left[{ }^{6}\right]$. В еще меньшей степени следует ожидать возникновения узких антипровалов в спектре $S_{1} \leftarrow S_{0}$-поглощения при возбуждении системы в другие электронные состояния. Как следует из общих соображений $\left[{ }^{7}\right]$ и как показано экспериментально для ряда систем $\left[{ }^{7-9}\right]$, корреляция между частотами переходов в разные электронные состояния в общем случае довольно слабая. Поэтому можно ожидать, что возбуждение перилена в высокие электронные состояния приводит к равномерному по неоднородному ансамблю заселению $S_{1}$-состояния даже при использовании монохроматического света. В таком случае не следует вообще ожидать существенного изменения спектра $S_{1} \leftarrow S_{0}$-поглощения, так как уменьшение поглощения в некотором спектральном интервале из-за выжигания компенсируется спектральным приходом в этот же интервал примесей, выжженных из других участков спектра.

Ситуация существенно меняется если учесть, что длинноволновое облучение приводит также к спектрально селективному опустошению $S_{1}$-состояния из-за светоиндуцированных переходов в колебательные состояния основного электронного состояния $\left[{ }^{10}\right]$. Индуцированное флуоресценцией опустошение возбужденного электронного состояния в кристаллах нафталина с примесью антрацена при сильном лазерном возбуждении было отмечено ранее $\left[{ }^{11}\right]$, а индуцированное лазерным облучением опустошение возбужденного электронного уровня было в $\left[{ }^{12}\right]$ использовано для определения дипольного момента электронного перехода молекулы антрацена в этой же системе.

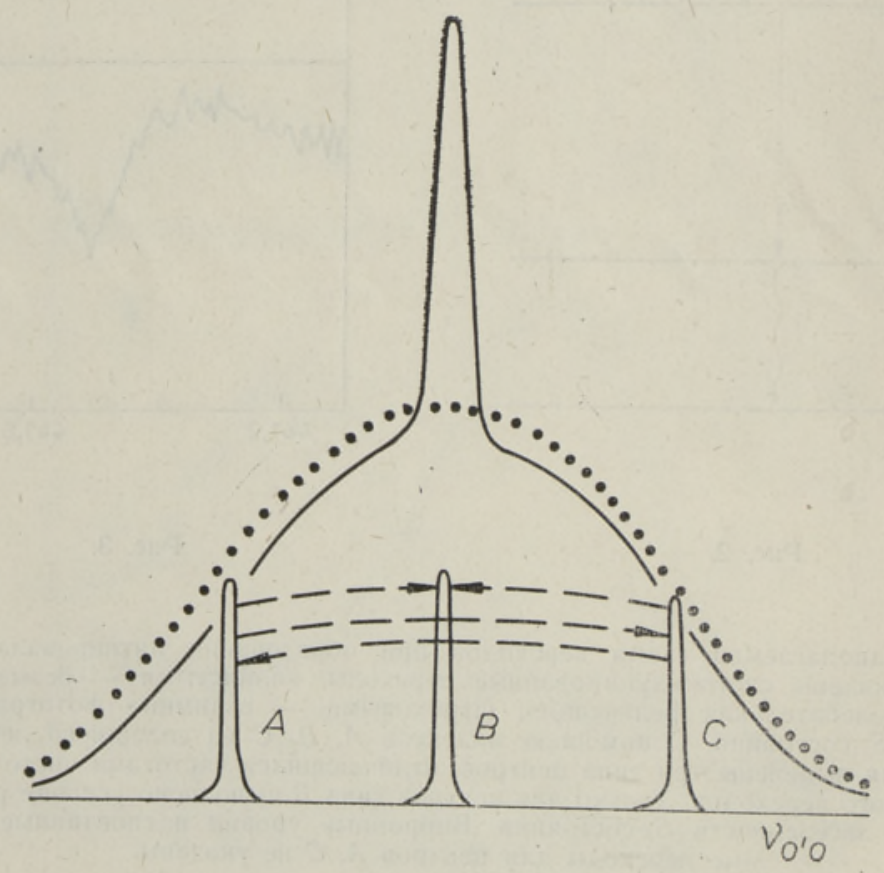

Рис. 4. Схема образования антипровала в функцин неоднородного распределения. Пунктиром указано исходное распределение. Линии $A, B, C$ - чисто электронные линии примесей, $S_{1}$-уровни которых выделены на рис. 2 , штриховыми стрелками указаны пере-
ходы, обусловленные фототрансформациями примесей. 
В нашем случае облучение на длинах волн 448,8 и 448,65 нм индуцирует переход $0^{\prime} \rightarrow 1$ по внутримолекулярному колебанию с частотой $355 \mathrm{~cm}^{-1}$ в молекулах перилена, у которых частота этого бесфононного перехода находится в резонансе со световым излучением (рис. 2). Такой спектрально селективный сброс заселенности возбужденного состояния нами наблюдался по возникновению динамического (краткоживущего) провала в спектре возбуждения при регистрации флуоресценции на вибронном $0^{\prime} \rightarrow 1\left(1370 \mathrm{~cm}^{-1}\right)$ переходе (рис. 3,б). Здесь использовались два световых импульса одновременно попадающих на объект. Первый импульс, длина волны которого сканировалась от 441 до 442 нм, осуществлял переход $0^{\prime} \leftarrow 0$, а второй на длине волны 448,5 нм индуцировал переход $0^{\prime} \rightarrow 1\left(355 \mathrm{~cm}^{-1}\right)$.

Допустим теперь, что молекулярные трансформации, приводящие к выжиганию провалов, происходят или в $S_{1}$-состоянии, как это изображено на рис. 2 , или в высоких $\left(>355 \mathrm{~cm}^{-1}\right)$ колебательных состояниях основного электронного состояния. В таком случае у молекул, в которых индуцируется переход $0^{\prime} \rightarrow 1\left(355 \mathrm{~cm}^{-1}\right)$, уменьшается вероятность выжигаться в единицу времени. В результате переход молекул в спектральный интервал, где осуществляется «сбрасывание», будет превышать их уход оттуда, что приводит к образованию антипровала в спектре (рис. 4).

Отметим, что наблюдение антипровалов, возникающих благодаря спектрально селективному торможению фототрансформации светоиндуцированным сбросом заселенности возбужденного состояния, открывает новые возможности для исследования колебательной релаксации и каналов фототрансформации в молекулярных примесях. В частности, ширина резонансного к «сбрасывающему» излучению антипровала в спектре флуоресценции позволяет определить однородную ширину соответствующей вибронной линии испускания, что невозможно или трудно выполнить другими методами.

Авторы благодарны К. К. Ребане за обсуждение и полезные замечания.

\section{ЛИТ Е РА Т У РА}

1. Гороховский А. А., Каарли Р. К., Ребане Л. А. Письма в ЖЭТФ, 20, вып. 7, 474479 (1974).

2. Kharlamov, B. M., Personov, R. I., Bykovskaya, L. A. Opt. Commun., 12, № 1, 191-193 (1974).

3. Völker, S., Macfarlane, R. M. IBM J. Res. Develop., 23, № 5, 547-555 (1979).

4. Kikas, J., Rätsep, M. Phys. status solidi (b), 112, № 2, 409-415 (1982).

5. Olson, R. W., Lee, H. W. H., Patterson, F. G., Fayer, M. D., Shelby, R. M., Purum, D. P., Macfarlane, R. M. J. Chem. Phys,, 77, № 5, 2283-2289 (1982).

6. Харламов Б. М., Альици Е. И., Персонов Р. Н. Изв. АН СССР. Сер. физ., 48, № 7 , 1313-1321 (1984).

7. Саари П. М., Тамм Т. Б. Изв. АН СССР. Сер. физ., 39, № 11, 2321-2325 (1975).

8. Tamm, T. B., Saari P. M. Chem. Phys. Lett., 30, № 2, 219-222 (1975).

9. Al'shits, E. I., Personov, R. I., Kharlamov, B. M. Chem. Phys. Lett., 40, № 1, $116-120$ (1976).

10. Галанин М. Д., Кирсанов Б. М., Чижикова З. А. Письма в ЖЭТФ, 9, вып. 9, 502507 (1969).

11. Treshchalov, A. B., Sildos, I. R., Rozman, M. G. In: Proc. of the VIII Int. Conf. on Raman Spectr., Bordeaux, 1982, 189.

12. Treshchalov, A. B., Rozman, M. G. Opt. Commun., 47, № 4, 262-267 (1983).

Институт физики
Академии наук Әстонской ССР
Поступила в редакцию 25/IV 1984 


\section{PUSIVATE ANTIAUKUDE TEKE MITTEHOMOGEENSETES LISANDI- SPEKTRITES MITTERESONANTSEL LASERKIIRITUSEL}

On avastatud kitsaste (poollaius $0,1 \mathrm{~nm}$ ) püsivate antiaukude teke perüleeni ergastusspektris perüleeni ja etanooli tahkes lahuses $(T=4,2 \mathrm{~K})$ viimase kiiritamisel võimsa monokromaatse impulssvalgusega väljaspool lisandi neeldumisriba ning esitatud nähtuse võimalik mehhanism ja analüüsitud spektroskoopilisi rakendusi.

\section{SILDOS, J. KIKAS}

\section{FORMATION OF STABLE ANTIHOLES IN INHOMOGENEOUS IMPURITY SPECTRA ON NONRESONANT LASER IRRADIATION}

A formation of narrow $(0.1 \mathrm{~nm}$ halfwidth) stable antiholes in the excitation spectra of perylene-ethanol solid solution at $4.2 \mathrm{~K}$ on a high-power $\left(10 \mathrm{~kW} \cdot \mathrm{cm}^{-2}\right)$ monochromatic pulsed laser irradiation outside the perylene absorption band, is detected. A possible mechanism of this phenomenon involves a spectrally nonselective population of $S_{1}$ electronic state via two-photon transitions to higher electronic states and its selective depopulation via stimulated transitions to the vibronic levels of $S_{0}$ state, which are less active in the phototransformations. This results in spectral storage of impurities at the frequency of vibronic transition resonant to the exciting light. The phenomenon may be used for the studies of phototransformation pathways in impurity systems and for the determination of homogeneous linewidths of vibronic fluorescence lines. 